\title{
Perinatal Changes of 2,3-Diphosphoglycerate and Oxygen Affinity in Mammals Not Having Fetal Type Hemoglobins
}

\author{
HARRY BARD ${ }^{(2)}$ AND MARTIN SHAPIRO \\ Perinatal Service and Pediatric Research Center of Hopital Sainte Justine, Department of Pediatrics, University of \\ Monireal, Canada
}

\section{Sumnary}

The interrelationship of 2,3-diphosphoglycerate (2,3-DPG) and P50 levels during the fetal and postnatal life were determined in two mammalian species which do not have a switchover of hemoglobin type at the end of their fetal development. In the guinea pig and rabbit, the 2,3-DPG levels remain low during fetal life and increase only after birth remaining elevated throughout adult life. The adult levels were reached at 2 days of age in the guinea pig and the 20th day in rabbit. Fetal P50 values increased only after birth, paralleling the rise in the 2,3-DPG. The rapidity of the postnatal rise in 2,3-DPG and decrease in $P 50$ appears related to the maturity of the newborn animal at birth in these species.

\section{Speculation}

The synthesis of 2,3-DPG during fetal life appears to be limited. This limitation may be due to the lack of substrate or the key enzyme, 2,3-DPG mutase, during fetal development.

Hemoglobin function appears clearly adapted to meet the different metabolic needs of the fetus and the adult in their respective environments. Efficient oxygen transport requires that hemoglobin both combine with oxygen at prevailing pressures and release it to tissues at pressures appropriate for metabolism.

Fetal development in a variety of species is accompanied by a switchover from fetal to adult hemoglobin synthesis $(1,2)$. This switchover is part of a process that enables the fetal red blood cell to adapt to postnatal physiologic requirements. Another form of postnatal adaptation which occurs within the red blood cell is the neonatal increase in $2,3-\mathrm{DPG}$ synthesis $(6,7)$.

In order to further our understanding of the modulation of oxygen affinity along with the changes in 2,3-DPG during the adaptation from the intra- to the extrauterine environment, comparative studies were carried out in rabbits and guinea pigs during the perinatal period. These mammals were selected in order to evaluate the relationship of changes in oxygen affinity to the state of activity and maturation of the newborn animal. The goal was to determine pre- and postnatal changes in red blood cell oxygen affinity and concomitant levels of 2,3-DPG in both species.

The rabbit and guinea pig are similar in many respects in that both lack a specific fetal type hemoglobin (1 I), their hemoglobins interact with 2,3-DPG (5), the effect of the pH on the unloading of oxygen from their blood is within the same range (13), and they are similar in size (14). However, these species differ in their length of gestation and state of maturity at birth. Rabbits that require 30 days of gestation are born blind, deaf, hairless, and helpless, and are confined to a "nest" for about 3 weeks. In contrast, guinea pigs that have a gestational period of 68 days are well developed, possess a full haircoat, and can run about and fend for themselves soon after birth.

\section{MATERIALS AND METHODS}

New Zealand White rabbits and Hartley breed guinea pigs were used for this study (17). Fetal blood, pooled from littermates, was obtained after Cesarean section during the last trimester of the gestational period. Blood samples were also obtained, at different ages, during postnatal life.

Erythrocyte DPG concentration of fresh heparinized blood was determined using the stoichiometric conversion of DPG to glyceraldehyde-3-phosphate and oxidation of NADH to NAD, according to the method of Keitt (10), and was expressed as micromoles per gram of hemoglobin. The reagents were obtained from Sigma Chemical Company (18).

The P50 was determined by gas mixing tonometry using a IL 217 Blood Gas Laboratory (19) (213 blood gas analyser tonometer, 208 gas mixing system and 182 coximeter). Analyses were carried out at $37^{\circ}, \mathrm{pH} 7.40$, and $\mathrm{PCO}_{2}$ of $40 \mathrm{~mm} \mathrm{Hg}$. Samples of fresh blood were equilibrated in a tonometer for $30 \mathrm{~min}$ at $37^{\circ}$ with gas mixtures containing $40 \mathrm{~mm} \mathrm{Hg}( \pm 0.05 \mathrm{~mm} \mathrm{Hg}) \mathrm{CO}_{2}$ and varying proportions of $\mathrm{O}_{2}$ and $\mathrm{N}_{2}$. The tonometer permits equilibration and sampling of successive aliquots of blood at different $\mathrm{O}_{2}$ tensions. Measurement of $\mathrm{O}_{2}$ saturation, $\mathrm{pH}$, and $\mathrm{PO}_{2}$ provided the information for plotting the $\mathrm{O}_{2}$ dissociation curve and P50. $\mathrm{PO}_{2}$ was converted to values at pH 7,40 using a Bohr effect correction factor of 0.47 for the guinea pig and 0.45 for the rabbit (9). The oxygen saturation values obtained by use of the coximeter were verified by determining the oxygen content of blood samples equilibrated in the tonometer by use of the lex- $\mathrm{O}_{2}-\mathrm{Con}(20)$.

\section{RESULTS}

The changes in 2,3-DPG and P50 observed in rabbits during the period of the study are shown in Figure 1 . The mean prenatal 2,3-DPG level remained unchanged $(2.1 \pm 0.9)$ until after I week of age. After the first week, there was a gradual increase reaching adult levels at 20 days which were 10 times greater than the fetal 2,3-DPG levels. Parallel changes were observed in red blood cell oxygen affinity (prenatal P50 was $15 . \mathrm{I} \pm I .4 \mathrm{~mm} \mathrm{Hg}$ adult P50 was $27.2 \pm 3.9 \mathrm{~mm} \mathrm{Hg}$ ). There was a significant correlation between P50 and 2,3-DPG concentration $(r=0.95, P<0.001)$.

Figure 2 demonstrates the 2,3-DPG and P50 data obtained preand postnatally in guinea pigs. The mean prenatal level was unchanged $(5.1 \pm 1.9)$ until after the first $24 \mathrm{hr}$ after birth; there was then a 3-fold increase in 2,3-DPG. By 2 days of age, organic phosphate levels were within range of adult values $(18.0 \pm 3.8)$. The increase in P50 which was observed after the rise in 2,3-DPG (prenatal P50 was $16.1 \pm 1.2 \mathrm{~mm} \mathrm{Hg}$; adult P50 was $24.2 \pm 1.8$ $\mathrm{mm} \mathrm{Hg}$ ). There was a significant correlation between P50 and 2,3DPG concentration $(r=0.87, P<0.001)$.

\section{DISCUSSION}

The gestational period of the guinea pig averages 68 days which is unusually long for a rodent. The newborn guinea pig is well 


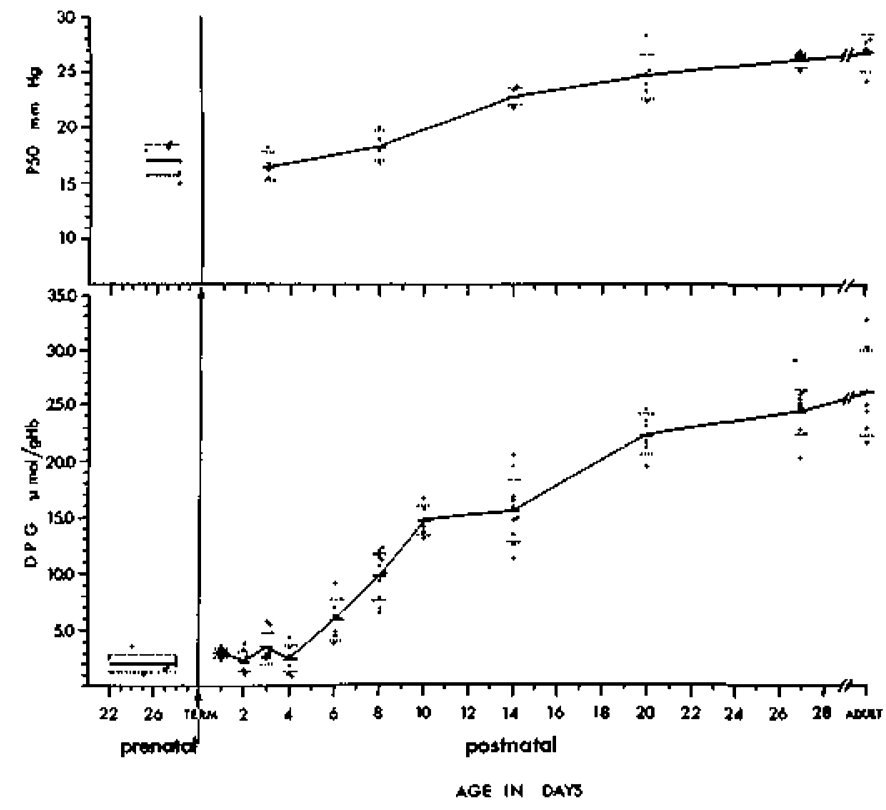

Fig. 1. Mean (-) and SD (...) of the changes of both 2,3-DPG and $\mathbf{P 5 0}$ in relation to pre- and postnatal age in the rabbit. Solid line unites the mean values obtained from 4-10 determinations for each age.

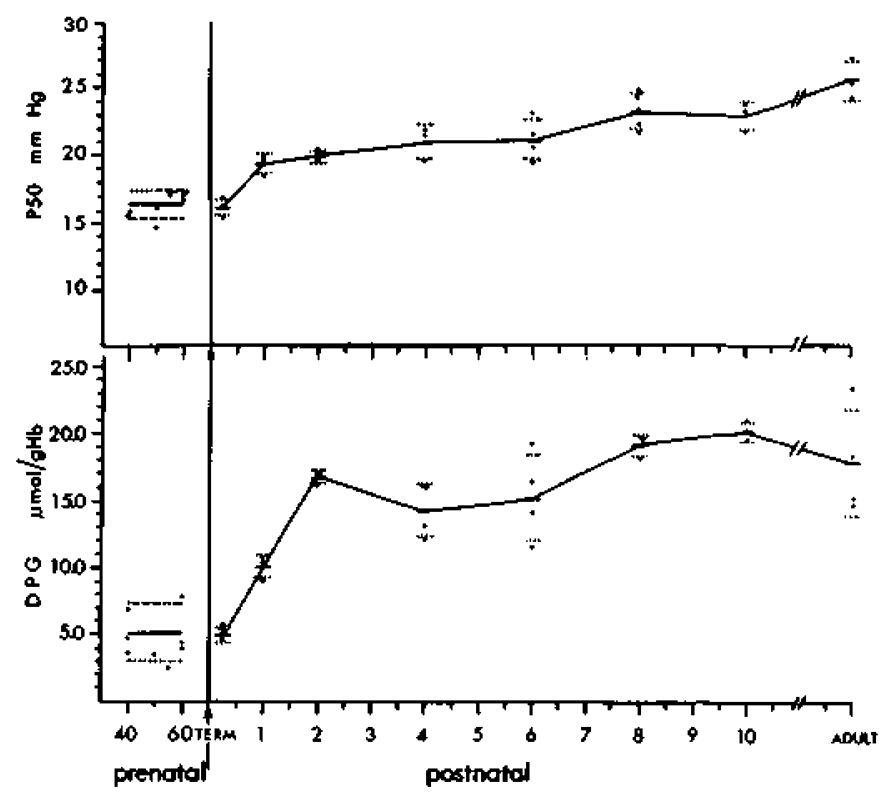

AGE IN DAYS

Fig. 2. Mean (-) and SD (...) of the changes of both 2,3-DPG and P50 in relation to pre- and postnatal age in the guinea pig. Solid line unites the mean values obtained from 3-10 determinations for each age.

developed, possesses a full haircoat, and is able to walk and fend for itself. In contrast, the gestational period of the rabbit is $30-32$ days and the newborn rabbit is decidedly immature-born blind, deaf, hairless, and helpless - and does not open its eyes until about 10-12 days.

In the two species compared, their fetuses do not have a switchover of hemoglobin type synthesis in the last trimester of gestation. Their changes of oxygen affinity during the postnatal period seem to be dependent upon the increase in red blood cell 2,3-DPG. They do however have differences in meonatal maturity and postnatal activity. The 2,3-PG levels appear to be constant during the fetal life studied in both species and the rate at which 2,3-DPG levels increase after birth appeared to be directly related to the maturity of the newborn and its degree of activity. There must be a far greater demand for oxygen for movement and heat production in the newborn guinea pig than in the newborn rabbit. A more rapid decrease in oxygen affinity soon after birth should provide an increase in oxygen available to the tissues to facilitate these physiologic requirements.

A variety of other newborn mammalian species which do not have a switchover of hemoglobin type at the end of their fetal development have been similarly investigated. These include pig (7), dog (8), horse (6), and opossum (12). They all exhibit a lower 2,3-DPG concentration and a lower P50 at birth than during adult life. During their postnatal development they demonstrate a decrease in oxygen affinity corresponding to an increase in red blood cell 2,3-DPG. In the horse, fetal 2,3-DPG does not change during the latter half of gestation, but, within 5 days after birth, adult levels are reached. In the pig, the increase in 2,3-DPG is 5-fold by 17 days, at which time $\mathrm{P} 50$ and 2,3-DPG approach adult values. The newborn dog demonstrates a steady increase in P50 and 2,3DPG from birth until 50-65 days of age.

The rapidity of postnatal increases in 2,3-DPG and P50 in the above species appears to be related to their physiologic maturation at birth. Relationships between the state of maturity of the newborn and oxygen affinity can best be shown by the postnatal changes in blood respiratory characteristics in the American opossum (12). The newborn opossum is very immature at birth, is confined to the maternal pouch, and is attached to the teat for the first 70 days after birth. The newborn leaves the pouch at 100 days. In these animals, there is a stepwise increase in P50 and 2,3DPG which is related to each stage of maturation, peaking at 148 days.

The postnatal decrease in oxygen affinity of mammalian blood can be described according to three distinct mechanisms:

1. There is a switchover from fetal to adult hemoglobin synthesis during the perinatal period (2), the adult-type hemoglobin having an intrinsically lower oxygen affinity. This is found in sheep (3) and goat (4). However, there is a transient 2,3-DPG rise in these animals during the neonatal period, which results in a decrease in fetal red blood cell oxygen affinity due to a lowering of red blood cell $\mathrm{pH}(3)$.

2. There is a switchover from fetal to adult hemoglobin synthesis during the perinatal period (l), the adult-type hemoglobin being affected by 2,3-DPG to a greater degree than fetal hemoglobin. This is found in humans (16) and monkeys (15) where the differences in the affinities of fetal and adult hemoglobin for 2,3-DPG can account for the postnatal decrease in red blood cell oxygen affinity.

3. Levels of fetal red blood cell 2,3-DPG are low and then increase during the postnatal period without any changes in hemoglobin type. Examples include the animals investigated in the present study.

\section{CONCLUSION}

This study demonstrates the interrelationship of 2,3-DPG and P50 in mammals not having a switchover of hemoglobin type near the end of gestation and, also, that there is a direct relationship between the state of maturity at birth and the rapidity of the postnatal decrease of oxygen affinity. This would suggest that there are mechanisms which carefully regulate $2,3-\mathrm{DPG}$ levels during the perinatal period in these animals. Because of the remarkable changes of 2,3-DPG levels found during the perinatal period, they are excellent experimental models to study the mechanisms that stimulate and control 2,3-DPG synthesis.

\section{REFERENCES AND NOTES}

I. Bard, H.: The postnatal decline of hemoglobin F synthesis in normal full-term infants. J. Clin. Invest. 55: 395 (1975).

2. Bard. H Battaglia, F, C Makowski, E L and Meschia, $G$. The synthesis of fetal and adult hemoglobin in sheep during the perinatal period. Proc. Soc. Exp. Biol. Med., /39: 1148 (1972).

3. Battaglia, F. C., McGaughey, H., Makowski, E. L., and Meschia, G.: Postnatal 